\title{
A Characterization of a Two-Weight Inequality for Discrete Two-Dimensional Hardy Operators
}

\author{
Y. Rakotondratsimba
}

\begin{abstract}
A bstract. We obtain a characterization of non-negative double sequences $\mathcal{V}=\left(\mathcal{V}\left(n_{1}, n_{2}\right)\right)_{n_{1} ; n_{2}}$ and $\mathcal{U}=\left(\mathcal{U}\left(n_{1}, n_{2}\right)\right)_{n_{1}, n_{2}}$ for which the two-dimensional discrete Hardy operator $\mathbf{H}$ is bounded from $\ell^{p}(\mathcal{V})$ into $\ell^{q}(\mathcal{U})$ whenever $1<p \leq q<\infty$.
\end{abstract}

Keywords: Inequalities, weights, discrete Hardy operators

AMS subject classification: 26 D 15

\section{Introduction}

The discrete two-dimensional Hardy operator is defined by

$$
(\mathbf{H} \mathcal{F})\left(n_{1}, n_{2}\right)=\sum_{i_{1}=0}^{n_{1}} \sum_{i_{2}=0}^{n_{2}} \mathcal{F}_{i_{1} i_{2}} \quad\left(n_{1}, n_{2} \in \mathbb{N}_{0}\right)
$$

For the sake of simplicity and as in the continuous case, the non-negative double sequence $\mathcal{F}_{i_{1} i_{2}}$ is merely denoted by $\mathcal{F}\left(i_{1}, i_{2}\right)$.

Our purpose in this work is to derive necessary and sufficient condition on nonnegative double sequences $\mathcal{U}\left(n_{1}, n_{2}\right)$ and $\mathcal{V}\left(n_{1}, n_{2}\right)$ for which there is a constant $C>0$ such that

$$
\left(\sum_{n_{1}=0}^{\infty} \sum_{n_{2}=0}^{\infty}(\mathbf{H} \mathcal{F})^{q}\left(n_{1}, n_{2}\right) \mathcal{U}\left(n_{1}, n_{2}\right)\right)^{\frac{1}{q}} \leq C\left(\sum_{n_{1}=0}^{\infty} \sum_{n_{2}=0}^{\infty} \mathcal{F}^{p}\left(n_{1}, n_{2}\right) \mathcal{V}\left(n_{1}, n_{2}\right)\right)^{\frac{1}{p}}
$$

for all double sequences $\mathcal{F}(\cdot, \cdot) \geq 0$ and with $1<p \leq q<\infty$. As in the continuous setting, $\mathcal{U}(\cdot, \cdot)$ and $\mathcal{V}(\cdot, \cdot)$ will be called weights. As a rule, the boundedness (1.1) will be also denoted by $\mathbf{H}: \ell_{v}^{p} \rightarrow \ell_{u}^{q}$.

Inequality (1.1) is involved in many parts of Analysis as in questions of convergence and summability of double series, and in analysis of random walks on infinite graphs [2, $5,7]$.

Y. Rakotondratsimba: Institut Polytechnique St.-Louis, E.P.M.I., 13 bd de I'Hautil, 95092 Cergy-Pontoise cedex, France 
According to B. Pachpatte [7], the boundedness $\mathbf{H}: \ell_{\mathcal{V}}^{p} \rightarrow \ell_{u}^{p}$ is true for $\mathcal{V}\left(n_{1}, n_{2}\right)=$ 1 and $\mathcal{U}\left(n_{1}, n_{2}\right)=n_{1}^{-p} n_{2}^{-p}$ with $1<p<\infty$. Later D. Y. Hwang [2] proved that $\mathbf{H}: \ell_{\nu}^{p} \rightarrow$ $\ell_{U}^{p}$ whenever $\mathcal{U}\left(n_{1}, n_{2}\right)=\lambda_{1}\left(n_{1}\right) \lambda_{2}\left(n_{2}\right)\left[\lambda_{1}(1)+\ldots+\lambda_{1}\left(n_{1}\right)\right]^{-p}\left[\lambda_{2}(1)+\ldots+\lambda_{2}\left(n_{2}\right)\right]^{-p}$ and $\mathcal{V}\left(n_{1}, n_{2}\right)=\left[\lambda_{1}\left(n_{1}\right) \lambda_{2}\left(n_{2}\right)\right]^{1-p}$ with $\lambda_{1}\left(n_{1}\right)$ and $\lambda_{2}\left(n_{2}\right)>0$. So for this case the inequality in question is a two-dimensional version of the well-known Copson inequality

$$
\sum_{k=1}^{\infty}\left[\sum_{l=1}^{k} \lambda_{l} \varphi(l)\right]^{p} \frac{\lambda_{k}}{\left[\lambda_{1}+\ldots+\lambda_{k}\right]^{p}} \leq\left(\frac{p}{p-1}\right)^{p} \sum_{k=1}^{\infty} \varphi^{p}(k) \lambda_{k} \quad(\varphi(k) \geq 0) .
$$

Recently Z. Németh [6] proved that $\mathbf{H}: \ell_{\mathcal{V}}^{p} \rightarrow \ell_{\mathcal{V}}^{p}$ whenever $\mathcal{V}\left(n_{1}, n_{2}\right)=v_{1}\left(n_{1}\right) v_{2}\left(n_{2}\right)$ and $v_{1}\left(n_{1}\right), v_{2}\left(n_{2}\right)$ are quasi-geometrically decreasing.

For all of these quoted results in $[2,6,7]$ the weights are of product types in the sense that $\mathcal{V}\left(n_{1}, n_{2}\right)=v_{1}\left(n_{1}\right) v_{2}\left(n_{2}\right)$ and $\mathcal{U}\left(n_{1}, n_{2}\right)=u_{1}\left(n_{1}\right) u_{2}\left(n_{2}\right)$. These twodimensional results with weights of product types are actually consequences of the one-dimensional ones [4]. And the crux of the matter is about weights not of product types like $\mathcal{W}\left(n_{1}, n_{2}\right)=\left(n_{1}+n_{2}\right) 3^{-\left(n_{1}+n_{2}\right)}$ and $\mathcal{W}\left(n_{1}, n_{2}\right)=\left(n_{1}+n_{2}\right)^{-\left(n_{1}+n_{2}\right)}$. A first investigation in this direction was done by $A$. Kamaly and the author in [4].

In this work we find a necessary and sufficient condition on weights $\mathcal{U}(\cdot, \cdot)$ and $\mathcal{V}(\cdot, \cdot)$ for which $\mathbf{H}: \ell_{\mathcal{V}}^{p} \rightarrow \ell_{\mathcal{U}}^{q}$. Indeed, until this paper, a characterization result seems not available in the literature. However, the analogue integral inequality

$$
\begin{gathered}
\left(\int_{0}^{\infty} \int_{0}^{\infty}\left[\int_{0}^{x_{1}} \int_{0}^{x_{2}} f\left(y_{1}, y_{2}\right) d y_{1} d y_{2}\right]^{q} u\left(x_{1}, x_{2}\right) d x_{1} d x_{2}\right)^{\frac{1}{q}} \quad(\forall f(\cdot, \cdot) \geq 0) \\
\leq C\left(\int_{0}^{\infty} \int_{0}^{\infty} f^{p}\left(x_{1}, x_{2}\right) v\left(x_{1}, x_{2}\right) d x_{1} d x_{2}\right)^{\frac{1}{p}}
\end{gathered}
$$

was already solved fifteen years ago by E. Sawyer [8]. Consequently, the present work aims to fill this gap between the discrete inequality (1.1) and its continuous version (1.2). To derive the characterization for $\mathbf{H}: \ell_{\mathcal{V}}^{p} \rightarrow \ell_{u}^{q}$, as in [3] (see Section 3), our idea will make use of the fact that there is some equivalence between (1.1) and (1.2) for some judicious choice of the weights $u(\cdot, \cdot)$ and $v(\cdot, \cdot)$.

Our results are stated in the next Section 2, and their proofs will be performed in Section 3.

\section{The results}

Throughout this paper it is always assumed that

$$
1<p \leq q<\infty, \quad p^{\prime}=\frac{p}{p-1}, \quad q^{\prime}=\frac{q}{q-1}
$$

and

$$
\mathcal{U}\left(n_{1}, n_{2}\right), \mathcal{V}\left(n_{1}, n_{2}\right) \text { are weight functions defined on } \mathbb{N}_{0}^{2} .
$$

To simplify and avoid some inconsistencies, it will be supposed that $\mathcal{V}\left(n_{1}, n_{2}\right) \neq 0$. 
Recall that our purpose in this work is to derive a characterization of weights $\mathcal{U}\left(n_{1}, n_{2}\right)$ and $\mathcal{V}\left(n_{1}, n_{2}\right)$ for which $\mathbf{H}: \ell_{V}^{p} \rightarrow \ell_{\mathcal{U}}^{q}$. This last means that for some constant $C>0$

$$
\left(\sum_{n_{1}=0}^{\infty} \sum_{n_{2}=0}^{\infty}(\mathbf{H} \mathcal{F})^{q}\left(n_{1}, n_{2}\right) \mathcal{U}\left(n_{1}, n_{2}\right)\right)^{\frac{1}{q}} \leq C\left(\sum_{n_{1}=0}^{\infty} \sum_{n_{2}=0}^{\infty} \mathcal{F}^{p}\left(n_{1}, n_{2}\right) \mathcal{V}\left(n_{1}, n_{2}\right)\right)^{\frac{1}{p}}
$$

for all double sequences $\mathcal{F}(\cdot, \cdot) \geq 0$.

Our main result reads as follows.

Theorem 2.1. Suppose that $\mathbf{H}: \ell_{\mathcal{V}}^{p} \rightarrow \ell_{\mathcal{U}}^{q}$. Then for some constant $A>0$ the three conditions

$$
\begin{gathered}
\left(\sum_{n_{1}=N_{1}}^{\infty} \sum_{n_{2}=N_{2}}^{\infty} \mathcal{U}\left(n_{1}, n_{2}\right)\right)^{\frac{1}{q}}\left(\sum_{m_{1}=0}^{N_{1}} \sum_{m_{2}=0}^{N_{2}} \mathcal{V}^{1-p^{\prime}}\left(m_{1}, m_{2}\right)\right)^{\frac{1}{p^{\prime}}} \\
\leq A \text { for all integers } N_{1}, N_{2} \geq 0 \\
\left(\int_{0}^{R_{1}} \int_{0}^{R_{2}}\left[\int_{0}^{x_{1}} \int_{0}^{x_{2}} v^{1-p^{\prime}}\left(y_{1}, y_{2}\right) d y_{1} d y_{2}\right]^{q} u\left(x_{1}, x_{2}\right) d x_{1} d x_{2}\right)^{\frac{1}{q}} \\
\leq A\left(\int_{0}^{R_{1}} \int_{0}^{R_{2}} v^{1-p^{\prime}}\left(x_{1}, x_{2}\right) d x_{1} d x_{2}\right)^{\frac{1}{p}} \text { for all } R_{1}, R_{2}>0 \\
\left(\int_{R_{1}}^{\infty} \int_{R_{2}}^{\infty}\left[\int_{x_{1}}^{\infty} \int_{x_{2}}^{\infty} u\left(y_{1}, y_{2}\right) d y_{1} d y_{2}\right]^{p^{\prime}} v^{1-p^{\prime}}\left(x_{1}, x_{2}\right) d x_{1} d x_{2}\right)^{\frac{1}{p^{\prime}}} \\
\leq A\left(\int_{R_{1}}^{\infty} \int_{R_{2}}^{\infty} u\left(x_{1}, x_{2}\right) d x_{1} d x_{2}\right)^{\frac{1}{q^{\prime}}} \text { for all } R_{1}, R_{2}>0
\end{gathered}
$$

are satisfied with weights $u\left(x_{1}, x_{2}\right)$ and $v\left(x_{1}, x_{2}\right)$ defined by

$$
\begin{aligned}
& u\left(x_{1}, x_{2}\right)=\sum_{m_{1}=0}^{\infty} \sum_{m_{2}=0}^{\infty} \mathcal{U}\left(m_{1}, m_{2}\right) \mathbb{I}_{\left[m_{1}, m_{1}+1\right) \times\left[m_{2}, m_{2}+1\right)}\left(x_{1}, x_{2}\right) \\
& v\left(x_{1}, x_{2}\right)=\sum_{m_{1}=0}^{\infty} \sum_{m_{2}=0}^{\infty} \mathcal{V}\left(m_{1}, m_{2}\right) \mathbb{I}_{\left[m_{1}, m_{1}+1\right) \times\left(m_{2}, m_{2}+1\right)}\left(x_{1}, x_{2}\right)
\end{aligned}
$$

Here $\mathbb{I}_{\left[m_{1}, m_{1}+1\right) \times\left(m_{2}, m_{2}+1\right)}$ denotes the characteristic function of the rectangle $\left[m_{1}, m_{1}+\right.$ 1) $\times\left[m_{2}, m_{2}+1\right)$. And actually in these conditions, $A=C$ provided that $C$ is the constant involved in (2.1).

Conversely, the boundedriess $\mathbf{H}: \ell_{v}^{p} \rightarrow \ell_{u}^{q}$ does hold whenever all conditions (2.2) (2.4) are satisfied. Precisely, (2.1) remains true with $C=c(p, q) A$ where $c(p, q)>0$ depends only on the indexes $p$ and $q$. 
However, it is still an open problem whether conditions (2.3) and (2.4) respectively can be replaced by

$$
\begin{aligned}
& \left(\sum_{n_{1}=0}^{N_{1}} \sum_{n_{2}=0}^{N_{2}}\left[\sum_{m_{1}=0}^{n_{1}} \sum_{m_{2}=0}^{n_{2}} \mathcal{V}^{1-p^{\prime}}\left(m_{1}, m_{2}\right)\right]^{q} \mathcal{U}\left(n_{1}, n_{2}\right)\right)^{\frac{1}{q}} \\
& \quad \leq A\left(\sum_{m_{1}=0}^{N_{1}} \sum_{m_{2}=0}^{N_{2}} \mathcal{V}^{1-p^{\prime}}\left(m_{1}, m_{2}\right)\right)^{\frac{1}{p}} \text { for all integers } N_{1}, N_{2} \geq 0
\end{aligned}
$$

and

$$
\begin{aligned}
& \left(\sum_{n_{1}=N_{1}}^{\infty} \sum_{n_{2}=N_{2}}^{\infty}\left[\sum_{m_{1}=n_{1}}^{\infty} \sum_{m_{2}=n_{2}}^{\infty} \mathcal{U}\left(m_{1}, m_{2}\right)\right]^{p^{\prime}} \mathcal{V}^{1-p^{\prime}}\left(n_{1}, n_{2}\right)\right)^{\frac{1}{p^{\prime}}} \\
& \quad \leq A\left(\sum_{m_{1}=N_{1}}^{\infty} \sum_{m_{2}=N_{2}}^{\infty} \mathcal{U}\left(m_{1}, m_{2}\right)\right)^{\frac{1}{q^{\prime}}} \text { for all integers } N_{1}, N_{2} \geq 0 .
\end{aligned}
$$

Although a characterization result is theoretically of great interest, it would be noted that a necessary and sufficient condition as the one obtained in Theorem 2.1 is not in general easy to handle in explicit computations. It means that other investigations on sufficient conditions (easily computable) for $\mathbf{H}: \ell_{v}^{p} \rightarrow \ell_{u}^{q}$ deserve to be done. This will be achieved by the author in a forthcoming paper.

For many problems in analysis in product spaces, it is useful to consider variants of the operator $\mathbf{H}$ like

$$
\begin{aligned}
& \left(\mathbf{H}_{* *} \mathcal{F}\right)\left(n_{1}, n_{2}\right)=\sum_{i_{1}=n_{1}}^{\infty} \sum_{i_{2}=n_{2}}^{\infty} \mathcal{F}\left(i_{1}, i_{2}\right) \\
& \left(\mathbf{H}_{1 *} \mathcal{F}\right)\left(n_{1}, n_{2}\right)=\sum_{i_{1}=0}^{n_{1}} \sum_{i_{2}=n_{2}}^{\infty} \mathcal{F}\left(i_{1}, i_{2}\right) \\
& \left(\mathbf{H}_{* 1} \mathcal{F}\right)\left(n_{1}, n_{2}\right)=\sum_{i_{1}=n_{1}} \sum_{i_{2}=0}^{n_{2}} \mathcal{F}\left(i_{1}, i_{2}\right) .
\end{aligned}
$$

A characterization for $\mathbf{H}_{* *}: \ell_{\mathcal{V}}^{p} \rightarrow \ell_{\mathcal{U}}^{q}$ can be immediately obtained by using the above Theorem 2.1. Indeed, by duality arguments, the former boundedness is equivalent to $\mathbf{H}: \ell_{\mathcal{V}_{1}}^{p_{1}} \rightarrow \ell_{u_{1}}^{q_{1}}$ where $p_{1}=q^{\prime}, q_{1}=p^{\prime}$ (so $p_{1} \leq q_{1}$ ) and $\mathcal{V}_{1}\left(n_{1}, n_{2}\right)=\mathcal{U}^{1-q^{\prime}}\left(n_{1}, n_{2}\right)$, $\mathcal{U}_{1}\left(n_{1}, n_{2}\right)=\mathcal{V}^{1-p^{\prime}}\left(n_{1}, n_{2}\right)$.

Our next result is related to the boundedness $H_{1 *}: \ell_{\mathcal{V}}^{p} \rightarrow \ell_{u}^{q}$.

Theorem 2.2. Suppose that $\mathbf{H}_{1 *}: \ell_{\mathcal{V}}^{p} \rightarrow \ell_{U}^{q}$. Then for some constant $A>0$ the three conditions

$$
\begin{gathered}
\left(\sum_{n_{1}=N_{1}}^{\infty} \sum_{n_{2}=0}^{N_{2}} \mathcal{U}\left(n_{1}, n_{2}\right)\right)^{\frac{1}{q}}\left(\sum_{m_{1}=0}^{N_{1}} \sum_{m_{2}=N_{2}}^{\infty} \mathcal{V}^{1-p^{\prime}}\left(m_{1}, m_{2}\right)\right)^{\frac{1}{p^{\prime}}} \\
\leq A \text { for all integers } N_{1}, N_{2} \geq 0
\end{gathered}
$$




$$
\begin{aligned}
& \left(\int_{0}^{R_{1}} \int_{R_{2}}^{\infty}\left[\int_{0}^{x_{1}} \int_{x_{2}}^{\infty} v^{1-p^{\prime}}\left(y_{1}, y_{2}\right) d y_{1} d y_{2}\right]^{q} u\left(x_{1}, x_{2}\right) d x_{1} d x_{2}\right)^{\frac{1}{q}} \\
& \leq A\left(\int_{0}^{R_{1}} \int_{R_{2}}^{\infty} v^{1-p^{\prime}}\left(x_{1}, x_{2}\right) d x_{1} d x_{2}\right)^{\frac{1}{p}} \text { for all } R_{1}, R_{2}>0 \\
& \left(\int_{R_{1}}^{\infty} \int_{0}^{R_{2}}\left[\int_{x_{1}}^{\infty} \int_{0}^{x_{2}} u\left(y_{1}, y_{2}\right) d y_{1} d y_{2}\right]^{p^{\prime}} v^{1-p^{\prime}}\left(x_{1}, x_{2}\right) d x_{1} d x_{2}\right)^{\frac{1}{p^{\prime}}} \\
& \leq A\left(\int_{R_{1}}^{\infty} \int_{0}^{R_{2}} u\left(x_{1}, x_{2}\right) d x_{1} d x_{2}\right)^{\frac{1}{q}} \text { for all } R_{1}, R_{2}>0
\end{aligned}
$$

are satisfied with weights $u\left(x_{1}, x_{2}\right)$ and $v\left(x_{1}, x_{2}\right)$ defined as in (2.5) and (2.6). And actually, in these conditions, $A=C$ provided that $C$ is the constant involved in $\mathrm{H}_{1 *}$ : $\ell_{v}^{p} \rightarrow \ell_{u}^{q}$.

Conversely, the boundedness $\mathrm{H}_{1 *}: \ell_{\nu}^{p} \rightarrow \ell_{u}^{q}$ does hold whenever all the conditions (2.9)-(2.11) are satisfied. Precisely, the constant involved in the boundedness takes the form $C=c(p, q) A$ where $c(p, q)>0$ only depends on the indexes $p$ and $q$.

A characterization for $\mathbf{H}_{* 1}: \ell_{\mathcal{V}}^{p} \rightarrow \ell_{\mathcal{u}}^{q}$ can be deduced (by duality argument) from this last result, since this boundedness is equivalent to $\mathbf{H}_{1 *}: \ell_{\mathcal{V}_{1}}^{p_{1}} \rightarrow \ell_{\mathcal{U}_{1}}^{q_{1}}$ where $p_{1}, q_{1}$ and $\mathcal{U}_{1}\left(n_{1}, n_{2}\right), \mathcal{V}_{1}\left(n_{1}, n_{2}\right)$ are defined as above.

\section{Proofs of results}

In this section we will give the details of the proof of Theorem 2.1 and sketch the arguments for Theorem 2.2.

Proof of Theorem 2.1. For convenience the inequality

$$
\begin{aligned}
& \left(\int_{0}^{\infty} \int_{0}^{\infty}\left[\int_{0}^{x_{1}} \int_{0}^{x_{2}} f\left(y_{1}, y_{2}\right) d y_{1} d y_{2}\right]^{q} u\left(x_{1}, x_{2}\right) d x_{1} d x_{2}\right)^{\frac{1}{q}} \\
& \quad \leq C\left(\int_{0}^{\infty} \int_{0}^{\infty} f^{p}\left(x_{1}, x_{2}\right) v\left(x_{1}, x_{2}\right) d x_{1} d x_{2}\right)^{\frac{1}{p}} \text { for all } f(\cdot, \cdot) \geq 0
\end{aligned}
$$

will be denoted by $H: L_{v}^{p} \rightarrow L_{u}^{q}$. Our plan is to prove Theorem 2.1 as follows.

A) First we show that the boundedness $\mathbf{H}: \ell_{\mathcal{V}}^{p} \rightarrow \ell_{u}^{q}$ implies condition (2.2) and $H: L_{v}^{p} \rightarrow L_{u}^{q}$ where the weights $u\left(x_{1}, x_{2}\right)$ and $v\left(x_{1}, x_{2}\right)$ are defined from $\mathcal{U}\left(n_{1}, n_{2}\right)$ and $\mathcal{V}\left(n_{1}, n_{2}\right)$ as in identities (2.5) and (2.6). Thus conditions (2.3) and (2.4) follow readily from the boundedness $H: L_{v}^{p} \rightarrow L_{u}^{q}$ as it is well-known in [8]. Consequently, the necessary part in the theorem is verified.

B) Next we check that condition (2.2) implies

$$
\left(\int_{R_{1}}^{\infty} \int_{R_{2}}^{\infty} u\left(x_{1}, x_{2}\right) d x_{1} d x_{2}\right)^{\frac{1}{q}}\left(\int_{0}^{R_{1}} \int_{0}^{R_{2}} v^{1-p^{\prime}}\left(x_{1}, x_{2}\right) d x_{1} d x_{2}\right)^{\frac{1}{p^{\prime}}} \leq A
$$


for all $R_{1}, R_{2}>0$. Then, because of [8: Theorem 1], the boundedness $H: L_{v}^{p} \rightarrow L_{u}^{q}$ does hold under conditions (2.3), (2.4) and (3.2).

C) Finally, we prove that condition (2.2) and $H: L_{v}^{p} \rightarrow L_{u}^{q}$ lead to the boundedness $\mathbf{H}: \ell_{\mathcal{V}}^{p} \rightarrow \ell_{\mathcal{u}}^{q}$. Therefore steps B) and C) yield the proof of the sufficient part in the theorem.

Proof of Part A. Suppose that $\mathbf{H}: \ell_{v}^{p} \rightarrow \ell_{u}^{q}$. It means that inequality (2.1) is true for some constant $C>0$. Consider integers $N_{1}, N_{2} \geq 0$. Applying (2.1) for any non-negative (double) sequence $\mathcal{F}\left(n_{1}, n_{2}\right)$ whose support is $\left\{0, \ldots, N_{1}\right\} \times\left\{0, \ldots, N_{2}\right\}$, then

$$
\begin{gathered}
\left(\sum_{m_{1}=0}^{N_{1}} \sum_{m_{2}=0}^{N_{2}} \mathcal{F}\left(m_{1}, m_{2}\right)\right)\left(\sum_{n_{1}=N_{1}}^{\infty} \sum_{n_{2}=N_{2}}^{\infty} \mathcal{U}\left(n_{1}, n_{2}\right)\right)^{\frac{1}{q}} \\
\leq C\left(\sum_{m_{1}=0}^{N_{1}} \sum_{m_{2}=0}^{N_{2}} \mathcal{F}^{p}\left(m_{1}, m_{2}\right) \mathcal{V}\left(n_{1}, n_{2}\right)\right)^{\frac{1}{p}} .
\end{gathered}
$$

This last inequality implies immediately condition (2.2) (with $A=C$ ) by taking $\mathcal{F}\left(n_{1}\right.$, $\left.n_{2}\right)=\mathcal{V}^{1-p^{\prime}}\left(n_{1}, n_{2}\right)$ in its support $\left\{0, \ldots, N_{1}\right\} \times\left\{0, \ldots, N_{2}\right\}$ and by using the identity $\left(1-p^{\prime}\right) p+1=\left(1-p^{\prime}\right)$.

Next the boundedness $H: L_{v}^{p} \rightarrow L_{u}^{q}$ is implied by $\mathbf{H}: \ell_{\mathcal{V}}^{p} \rightarrow \ell_{u}^{q}$ as the following chain of computation shows:

$$
\begin{aligned}
& \int_{0}^{\infty} \int_{0}^{\infty}\left[\int_{0}^{x_{1}} \int_{0}^{x_{2}} f\left(y_{1}, y_{2}\right) d y_{1} d y_{2}\right]^{q} u\left(x_{1}, x_{2}\right) d x_{1} d x_{2} \\
& =\sum_{n_{1}=0}^{\infty} \sum_{n_{2}=0}^{\infty} \int_{n_{1}}^{n_{1}+1} \int_{n_{2}}^{n_{2}+1}\left[\int_{0}^{x_{1}} \int_{0}^{x_{2}} f\left(y_{1}, y_{2}\right) d y_{1} d y_{2}\right]^{q} u\left(x_{1}, x_{2}\right) d x_{1} d x_{2} \\
& \leq \sum_{n_{1}=0}^{\infty} \sum_{n_{2}=0}^{\infty}\left[\int_{0}^{n_{1}+1} \int_{0}^{n_{2}+1} f\left(y_{1}, y_{2}\right) d y_{1} d y_{2}\right]^{q}\left(\int_{n_{1}}^{n_{1}+1} \int_{n_{2}}^{n_{2}+1} u\left(x_{1}, x_{2}\right) d x_{1} d x_{2}\right) \\
& =\sum_{n_{1}=0}^{\infty} \sum_{n_{2}=0}^{\infty}\left[\sum_{m_{1}=0}^{n_{1}} \sum_{m_{2}=0}^{n_{2}} \mathcal{F}\left(m_{1}, m_{2}\right)\right]^{q} \mathcal{U}\left(n_{1}, n_{2}\right) \\
& \text { (by using (2.5) and setting } \left.\mathcal{F}\left(m_{1}, m_{2}\right)=\int_{m_{1}}^{m_{1}+1} \int_{m_{2}}^{m_{2}+1} f\left(y_{1}, y_{2}\right) d y_{1} d y_{2}\right) \\
& \leq C^{q}\left(\sum_{n_{1}=0}^{\infty} \sum_{n_{2}=0}^{\infty} \mathcal{F}^{p}\left(n_{1}, n_{2}\right) \mathcal{V}\left(n_{1}, n_{2}\right)\right)^{\stackrel{q}{p}} \\
& \text { (due to the boundedness } \mathrm{H}: \ell_{\nu}^{\mathrm{p}} \rightarrow \ell_{u}^{q} \text { with the constant } C>0 \text { ) } \\
& \leq C^{q}\left(\sum_{n_{1}=0}^{\infty} \sum_{n_{2}=0}^{\infty}\left(\int_{n_{1}}^{n_{1}+1} \int_{n_{2}}^{n_{2}+1} f^{p}\left(x_{1}, x_{2}\right) v\left(x_{1}, x_{2}\right) d x_{1} d x_{2}\right)\right. \\
& \left.\times \mathcal{V}\left(n_{1}, n_{2}\right)\left(\int_{n_{1}}^{n_{1}+1} \int_{n_{2}}^{n_{2}+1} v^{1-p^{\prime}}\left(y_{1}, y_{2}\right) d y_{1} d y_{2}\right)^{p-1}\right)^{\frac{q}{p}} \\
& \text { (by the definition of } \mathcal{F}\left(m_{1}, m_{2}\right) \text { and using the Holder inequality) }
\end{aligned}
$$




$$
\begin{aligned}
=C^{q} & \left(\int_{0}^{\infty} \int_{0}^{\infty} f^{p}\left(x_{1}, x_{2}\right) v\left(x_{1}, x_{2}\right) d x_{1} d x_{2}\right)^{\frac{q}{p}} \\
& \quad\left(\text { since } V\left(n_{1}, n_{2}\right)\left(\int_{n_{1}}^{n_{1}+1} \int_{n_{2}}^{n_{2}+1} v^{1-p^{\prime}}\left(y_{1}, y_{2}\right) d y_{1} d y_{2}\right)^{p-1}=1\right)
\end{aligned}
$$

Proof of Part B. To check inequality (3.2), let us consider $R_{1}, R_{2}>0$ such that $N_{1} \leq R_{1}<N_{1}+1$ and $N_{2} \leq R_{2}<N_{2}+1$ for some integers $N_{1}, N_{2} \geq 0$. Then condition (2.2), with the constant $A>0$, leads to the test inequality (3.2) with the same constant since

$$
\begin{aligned}
\int_{R_{1}}^{\infty} \int_{R_{2}}^{\infty} u\left(x_{1}, x_{2}\right) d x_{1} d x_{2} & \leq \int_{N_{1}}^{\infty} \int_{N_{2}}^{\infty} u\left(x_{1}, x_{2}\right) d x_{1} d x_{2} \\
& =\sum_{n_{1}=N_{1}}^{\infty} \sum_{n_{2}=N_{2}}^{\infty} \int_{n_{1}}^{n_{1}+1} \int_{n_{2}}^{n_{2}+1} u\left(x_{1}, x_{2}\right) d x_{1} d x_{2} \\
& =\sum_{n_{1}=N_{1}}^{\infty} \sum_{n_{2}=N_{2}}^{\infty} \mathcal{U}\left(n_{1}, n_{2}\right)
\end{aligned}
$$

and

$$
\begin{aligned}
\int_{0}^{R_{1}} \int_{0}^{R_{2}} v^{1-p^{\prime}}\left(x_{1}, x_{2}\right) d x_{1} d x_{2} & \leq \int_{0}^{N_{1}+1} \int_{0}^{N_{2}+1} v^{1-p^{\prime}}\left(x_{1}, x_{2}\right) d x_{1} d x_{2} \\
& =\sum_{n_{1}=0}^{N_{1}} \sum_{n_{2}=0}^{N_{2}} \int_{n_{1}}^{n_{1}+1} \int_{n_{2}}^{n_{2}+1} v^{1-p^{\prime}}\left(x_{1}, x_{2}\right) d x_{1} d x_{2} \\
& =\sum_{n_{1}=0}^{N_{1}} \sum_{n_{2}=0}^{N_{2}} \mathcal{V}^{1-p^{\prime}}\left(n_{1}, n_{2}\right) .
\end{aligned}
$$

Proof of Part C. First some remarks can be done. For instance, (2.2) implies that for some $A>0$

$$
\begin{aligned}
& \left(\sum_{n_{1}=N_{1}}^{\infty} \mathcal{U}\left(n_{1}, N_{2}\right)\right)^{\frac{1}{q}}\left(\sum_{m_{1}=0}^{N_{1}} \mathcal{V}^{1-p^{\prime}}\left(m_{1}, N_{2}\right)\right)^{\frac{1}{p^{\prime}}} \leq A \\
& \left(\sum_{n_{2}=N_{2}}^{\infty} \mathcal{U}\left(N_{1}, n_{2}\right)\right)^{\frac{1}{q}}\left(\sum_{m_{2}=0}^{N_{2}} \mathcal{V}^{1-p^{\prime}}\left(N_{1}, m_{2}\right)\right)^{\frac{1}{p^{\prime}}} \leq A
\end{aligned}
$$

for all $N_{1}, N_{2} \in \mathbb{N}_{0}$. It is well-known that condition (3.4) implies the one-dimensional discrete weighted Hady inequality

$$
\left(\sum_{n_{1}=0}^{\infty}\left[\sum_{m_{1}=0}^{n_{1}} \varphi_{1}\left(m_{1}\right)\right]^{q} \mathcal{U}\left(n_{1}, N_{2}\right)\right)^{\frac{1}{q}} \leq c_{0} A\left(\sum_{n_{1}=0}^{\infty} \varphi_{1}^{p}\left(n_{1}\right) \mathcal{V}\left(n_{1}, N_{2}\right)\right)^{\frac{1}{p}}
$$

for all $\varphi_{1}(\cdot) \geq 0$, where $c_{0}=c_{0}(p, q)=\left(1+\frac{q}{p^{\prime}}\right)^{\frac{1}{q}}\left(1+\frac{p^{\prime}}{q}\right)^{\frac{1}{p}}$. Similarly, from condition (3.5) then

$$
\left(\sum_{n_{2}=0}^{\infty}\left[\sum_{m_{2}=0}^{n_{2}} \varphi_{2}\left(m_{2}\right)\right]^{q} \mathcal{U}\left(N_{1}, n_{2}\right)\right)^{\frac{1}{q}} \leq c_{0} A\left(\sum_{n_{2}=0}^{\infty} \varphi_{2}^{p}\left(n_{2}\right) \mathcal{V}\left(N_{1}, n_{2}\right)\right)^{\frac{1}{p^{\prime}}}
$$


for all $\varphi_{2}(\cdot) \geq 0$.

Next we are now in the position to derive the boundedness $\mathbf{H}: \ell_{\mathcal{V}}^{p} \rightarrow \ell_{u}^{q}$ by using condition (2.2) and $H: L_{v}^{p} \rightarrow L_{u}^{q}$. To do this let us split things as

$$
\begin{aligned}
\sum_{n_{1}=0}^{\infty} \sum_{n_{2}=0}^{\infty}(\mathbf{H} \mathcal{F})^{q}\left(n_{1}, n_{2}\right) \mathcal{U}\left(n_{1}, n_{2}\right) \\
\quad=\sum_{n_{1}=0}^{\infty} \sum_{n_{2}=0}^{\infty}\left[\sum_{m_{1}=0}^{n_{1}} \sum_{m_{2}=0}^{n_{2}} \mathcal{F}\left(m_{1}, m_{2}\right)\right]^{q} \mathcal{U}\left(n_{1}, n_{2}\right) \\
=S_{1}+S_{2}+S_{3}+S_{4}
\end{aligned}
$$

with

$$
\begin{aligned}
& S_{1}=\mathcal{F}^{q}(0,0) \mathcal{U}(0,0) \\
& S_{2}=\sum_{n_{2}=0}^{\infty}\left[\sum_{m_{2}=0}^{n_{2}} \mathcal{F}\left(0, m_{2}\right)\right]^{q} \mathcal{U}\left(0, n_{2}\right) \\
& S_{3}=\sum_{n_{1}=0}^{\infty}\left[\sum_{m_{1}=0}^{n_{1}} \mathcal{F}\left(m_{1}, 0\right)\right]^{q} \mathcal{U}\left(n_{1}, 0\right) \\
& S_{4}=\sum_{n_{1}=1}^{\infty} \sum_{n_{2}=1}^{\infty}\left[\sum_{m_{1}=0}^{n_{1}} \sum_{m_{2}=0}^{n_{2}} \mathcal{F}\left(m_{1}, m_{2}\right)\right]^{q} \mathcal{U}\left(n_{1}, n_{2}\right) .
\end{aligned}
$$

Consequently, our task remains to estimate each of $S_{i} \quad(i \in\{1,2,3,4\})$ by a term like

$$
(c A)^{q}\left(\sum_{n_{1}=0}^{\infty} \sum_{n_{2}=0}^{\infty} \mathcal{F}^{p}\left(n_{1}, n_{2}\right) \mathcal{V}\left(n_{1}, n_{2}\right)\right)^{\frac{q}{p}}
$$

where $c>0$ is a constant which only depends on the indexes $p$ and $q$.

Estimate of $S_{1}$. The conclusion follows from condition (2.2) since

$$
\begin{aligned}
S_{1} & =\left(\mathcal{F}^{p}(0,0) \mathcal{V}(0,0)\right)^{\frac{q}{p}}\left[\mathcal{U}^{\frac{1}{q}}(0,0)\left(\mathcal{V}^{1-p^{\prime}}(0,0)\right)^{\frac{1}{p^{p}}}\right]^{q} \\
& \left.\leq A^{q}\left(\mathcal{F}^{p}(0,0) \mathcal{V}(0,0)\right)^{\frac{q}{p}} \quad \text { (by condition (2.2) with } N_{1}=N_{2}=0\right) \\
& \leq A^{q}\left(\sum_{n_{1}=0}^{\infty} \sum_{n_{2}=0}^{\infty} \mathcal{F}^{p}\left(n_{1}, n_{2}\right) \mathcal{V}\left(n_{1}, n_{2}\right)\right)^{\frac{q}{p}} .
\end{aligned}
$$
Indeed,

Estimate of $S_{2}$. The main point is the Hardy inequality (3.7) (with $N_{1}=0$ ).

$$
\begin{aligned}
S_{2} & =\sum_{n_{2}=0}^{\infty}\left[\sum_{m_{2}=0}^{n_{2}} \mathcal{F}\left(0, m_{2}\right)\right]^{q} \mathcal{U}\left(0, n_{2}\right) \\
& \leq\left(c_{0} A\right)^{q}\left(\sum_{n_{2}=0}^{\infty} \mathcal{F}^{p}\left(0, n_{2}\right) \mathcal{V}\left(0, n_{2}\right)\right)^{\frac{q}{p}} \\
& \leq\left(c_{0} A\right)^{q}\left(\sum_{n_{1}=0}^{\infty} \sum_{n_{2}=0}^{\infty} \mathcal{F}^{p}\left(n_{1}, n_{2}\right) \mathcal{V}\left(n_{1}, n_{2}\right)\right)^{\frac{q}{p}} .
\end{aligned}
$$


Estimate of $S_{3}$. As for $S_{2}$, the proof is based on the Hardy inequality (3.6) (with $N_{2}=0$ ). The details are left to the readers.

Estimate of $S_{4}$. It can be noted that for some constant $c_{1}>0$, which depends only on $q$, then

$$
S_{4}=\sum_{n_{1}=1}^{\infty} \sum_{n_{2}=1}^{\infty}\left[\sum_{m_{1}=0}^{n_{1}} \sum_{m_{2}=0}^{n_{2}} \mathcal{F}\left(m_{1}, m_{2}\right)\right]^{q} \mathcal{U}\left(n_{1}, n_{2}\right) \leq c_{1}\left(S_{41}+S_{42}+S_{43}+S_{44}\right)
$$

where

$$
\begin{aligned}
& S_{41}=\sum_{n_{1}=1}^{\infty} \sum_{n_{2}=1}^{\infty} \mathcal{F}^{q}\left(n_{1}, n_{2}\right) \mathcal{U}\left(n_{1}, n_{2}\right) \\
& S_{42}=\sum_{n_{1}=1}^{\infty} \sum_{n_{2}=1}^{\infty}\left[\sum_{m_{2}=0}^{n_{2}-1} \mathcal{F}\left(n_{1}, m_{2}\right)\right]^{q} \mathcal{U}\left(n_{1}, n_{2}\right) \\
& S_{43}=\sum_{n_{1}=1}^{\infty} \sum_{n_{2}=1}^{\infty}\left[\sum_{m_{1}=0}^{n_{1}-1} \mathcal{F}\left(m_{1}, n_{2}\right)\right]^{q} \mathcal{U}\left(n_{1}, n_{2}\right) \\
& S_{44}=\sum_{n_{1}=1}^{\infty} \sum_{n_{2}=1}^{\infty}\left[\sum_{m_{1}=0}^{n_{1}-1} \sum_{m_{2}=0}^{n_{2}-1} \mathcal{F}\left(m_{1}, m_{2}\right)\right]^{q} \mathcal{U}\left(n_{1}, n_{2}\right)
\end{aligned}
$$

The term $S_{41}$ can be estimated just by using condition (2.2) since

$$
\begin{aligned}
S_{41} & =\sum_{n_{1}=1}^{\infty} \sum_{n_{2}=1}^{\infty}\left(\mathcal{F}^{p}\left(n_{1}, n_{2}\right) \mathcal{V}\left(n_{1}, n_{2}\right)\right)^{\frac{q}{p}}\left[\mathcal{U}^{\frac{1}{q}}\left(n_{1}, n_{2}\right)\left(\mathcal{V}^{1-p^{\prime}}\left(n_{1}, n_{2}\right)\right)^{\frac{1}{p^{p}}}\right]^{q} \\
& \leq A^{q} \sum_{n_{1}=1}^{\infty} \sum_{n_{2}=1}^{\infty}\left(\mathcal{F}^{p}\left(n_{1}, n_{2}\right) \mathcal{V}\left(n_{1}, n_{2}\right)\right)^{\frac{q}{p}} \quad(\text { by }(2.2)) \\
& \left.\leq A^{q}\left(\sum_{n_{1}=0}^{\infty} \sum_{n_{2}=0}^{\infty} \mathcal{F}^{p}\left(n_{1}, n_{2}\right) \mathcal{V}\left(n_{1}, n_{2}\right)\right)^{\frac{q}{p}} \quad \text { (since } \geq 1\right)
\end{aligned}
$$

The estimate of $S_{42}$ is essentially based on the Hardy inequality (3.7). Indeed,

$$
\begin{aligned}
S_{42} & \leq \sum_{n_{1}=1}^{\infty} \sum_{n_{2}=0}^{\infty}\left[\sum_{m_{2}=0}^{n_{2}} \mathcal{F}\left(n_{1}, m_{2}\right)\right]^{q} \mathcal{U}\left(n_{1}, n_{2}\right) \\
& \left.\leq\left(c_{0} A\right)^{q} \sum_{n_{1}=1}^{\infty}\left(\sum_{n_{2}=0}^{\infty} \mathcal{F}^{p}\left(n_{1}, n_{2}\right) \mathcal{V}\left(n_{1}, n_{2}\right)\right)^{\frac{q}{p}} \quad \text { (by (3.7) with } N_{1}=n_{1}\right) \\
& \left.\leq\left(c_{0} A\right)^{q}\left(\sum_{n_{1}=0}^{\infty} \sum_{n_{2}=0}^{\infty} \mathcal{F}^{p}\left(n_{1}, n_{2}\right) \mathcal{V}\left(n_{1}, n_{2}\right)\right)^{\frac{q}{p}} \quad \text { (since } \frac{q}{p} \geq 1\right)
\end{aligned}
$$

The term $S_{43}$ can be bounded similarly as $S_{42}$ by using the Hardy inequality (3.6). The estimate of $S_{44}$ makes use of the boundedness $H: L_{v}^{p} \rightarrow L_{u}^{q}$, say with the constant $c_{2} A$ 
where $c_{2}>0$ depends only on the indexes $p$ and $q$. Indeed,

$$
\begin{aligned}
S_{44}= & \sum_{n_{1}=1}^{\infty} \sum_{n_{2}=1}^{\infty}\left[\sum_{m_{1}=0}^{n_{1}-1} \sum_{m_{2}=0}^{n_{2}-1} \mathcal{F}\left(m_{1}, m_{2}\right)\right]^{q} \int_{n_{1}}^{n_{1}+1} \int_{n_{2}}^{n_{2}+1} u\left(x_{1}, x_{2}\right) d x_{1} d x_{2} \\
& \left.\quad \text { by the definition of } u\left(x_{1}, x_{2}\right) \text { in }(2,5)\right) \\
= & \sum_{n_{1}=1}^{\infty} \sum_{n_{2}=1}^{\infty} \int_{n_{1}}^{n_{1}+1} \int_{n_{2}}^{n_{2}+1}\left[\sum_{m_{1}=0}^{n_{1}-1} \sum_{m_{2}=0}^{n_{2}-1}\right. \\
& \left.\int_{m_{1}}^{m_{1}+1} \int_{m_{2}}^{m_{2}+1} f\left(y_{1}, y_{2}\right) d y_{1} d y_{2}\right]^{q} u\left(x_{1}, x_{2}\right) d x_{1} d x_{2} \\
& \left(\text { where } f\left(y_{1}, y_{2}\right)=\sum_{k_{1}=0}^{\infty} \sum_{k_{2}=0}^{\infty} \mathcal{F}\left(k_{1}, k_{2}\right) \mathbf{I}_{\left.\left(k_{1}, k_{2}+1\right) \times\left(k_{2}, k_{2}+1\right)\left(y_{1}, y_{2}\right)\right)}\right. \\
= & \sum_{n_{1}=1}^{\infty} \sum_{n_{2}=1}^{\infty} \int_{n_{1}}^{n_{1}+1} \int_{n_{2}}^{n_{2}+1}\left[\int_{0}^{n_{1}} \int_{0}^{n_{2}} f\left(y_{1}, y_{2}\right) d y_{1} d y_{2}\right]^{q} u\left(x_{1}, x_{2}\right) d x_{1} d x_{2} \\
\leq & \int_{0}^{\infty} \int_{0}^{\infty}\left[\int_{0}^{x_{1}} \int_{0}^{x_{2}} f\left(y_{1}, y_{2}\right) d y_{1} d y_{2}\right]^{q} u\left(x_{1}, x_{2}\right) d x_{1} d x_{2} \\
\leq & \left(c_{2} A\right)^{q}\left(\int_{0}^{\infty} \int_{0}^{\infty} f^{p}\left(x_{1}, x_{2}\right) v\left(x_{1}, x_{2}\right) d x_{1} d x_{2}\right)^{\frac{q}{p}}\left(\text { since } H: L_{2}^{p}-L_{2}^{q}\right) \\
= & \left(c_{2} A\right)^{q}\left(\sum_{n_{1}=0}^{\infty} \sum_{n_{2}=0}^{\infty} \int_{n_{1}}^{n_{1}+1} \int_{n_{2}}^{n_{2}+1} f^{p}\left(x_{1}, x_{2}\right) v\left(x_{1}, x_{2}\right) d x_{1} d x_{2}\right)^{\frac{q}{p}} \\
= & \left(c_{2} A\right)^{q}\left(\sum_{n_{1}=0}^{\infty} \sum_{n_{2}=0}^{\infty} \mathcal{F}^{p}\left(n_{1}, n_{2}\right) \mathcal{V}\left(n_{1}, n_{2}\right)\right)^{\frac{q}{p}} \\
& \left(b y \text { the definitions of } f\left(x_{1}, x_{2}\right) \text { and } v\left(x_{1}, x_{2}\right)\right. \text { in (2.6)). }
\end{aligned}
$$

This way Theorem 2.1 is completely proved

Proof of Theorem 2.2. We will restrict to outline the main lines of the proof, since the arguments are the same as those used for Theorem 2.1. To do this, it can be noted that the boundedness $H_{1 *}: L_{v}^{p} \rightarrow L_{u}^{q}$, i.e.

$$
\begin{aligned}
& \left(\int_{0}^{\infty} \int_{0}^{\infty}\left[\int_{0}^{x_{1}} \int_{x_{2}}^{\infty} f\left(y_{1}, y_{2}\right) d y_{1} d y_{2}\right]^{q} u\left(x_{1}, x_{2}\right) d x_{1} d x_{2}\right)^{\frac{1}{q}} \\
& \quad \leq C\left(\int_{0}^{\infty} \int_{0}^{\infty} f^{p}\left(x_{1}, x_{2}\right) v\left(x_{1}, x_{2}\right) d x_{1} d x_{2}\right)^{\frac{1}{p}} \text { for all } f(\cdot, \cdot) \geq 0
\end{aligned}
$$

is equivalent to the three conditions

$$
\begin{aligned}
& \left(\int_{R_{1}}^{\infty} \int_{0}^{R_{2}} u\left(x_{1}, x_{2}\right) d x_{1} d x_{2}\right)^{\frac{1}{q}}\left(\int_{0}^{R_{1}} \int_{R_{2}}^{\infty} v^{1-p^{\prime}}\left(x_{1}, x_{2}\right) d x_{1} d x_{2}\right)^{\frac{1}{p^{\prime}}} \\
& \quad \leq A \text { for all } R_{1}, R_{2}>0
\end{aligned}
$$




$$
\begin{aligned}
& \left(\int_{0}^{R_{1}} \int_{R_{2}}^{\infty}\left[\int_{0}^{x_{1}} \int_{x_{2}}^{\infty} v^{1-p^{\prime}}\left(y_{1}, y_{2}\right) d y_{1} d y_{2}\right]^{q} u\left(x_{1}, x_{2}\right) d x_{1} d x_{2}\right)^{\frac{1}{q}} \\
& \leq A\left(\int_{0}^{R_{1}} \int_{R_{2}}^{\infty} v^{1-p^{\prime}}\left(x_{1}, x_{2}\right) d x_{1} d x_{2}\right)^{\frac{1}{p}} \text { for all } R_{1}, R_{2}>0 \\
& \left(\int_{R_{1}}^{\infty} \int_{0}^{R_{2}}\left[\int_{x_{1}}^{\infty} \int_{0}^{x_{2}} u\left(y_{1}, y_{2}\right) d y_{1} d y_{2}\right]^{p^{\prime}} v^{1-p^{\prime}}\left(x_{1}, x_{2}\right) d x_{1} d x_{2}\right)^{\frac{1}{p}} \\
& \leq A\left(\int_{R_{1}}^{\infty} \int_{0}^{R_{2}} u\left(x_{1}, x_{2}\right) d x_{1} d x_{2}\right)^{\frac{1}{q}} \text { for all } R_{1}, R_{2}>0 .
\end{aligned}
$$

The proof of Theorem 2.2 will be fulfilled after doing the following three steps.

A) First we have to show that the boundedness $\mathbf{H}_{1 *}: \ell_{v}^{p} \rightarrow \ell_{u}^{q}$ implies condition (2.9) and $H_{1 *}: L_{v}^{p} \rightarrow L_{u}^{q}$ where the weights $u\left(x_{1}, x_{2}\right)$ and $v\left(x_{1}, x_{2}\right)$ are defined from $\mathcal{U}\left(n_{1}, n_{2}\right)$ and $\mathcal{V}\left(n_{1}, n_{2}\right)$ as in (2.5) and (2.6). Thus conditions (2.10) and (2.11) follow readily from (3.11) and (3.12), respectively. These last conditions are implied by $H_{1}$ * : $L_{v}^{p} \rightarrow L_{u}^{q}$ as it is noted above. And consequently the necessary part in the Theorem is verified.

B) Next we have to check that condition (2.9) implies (3.10). Consequently, the boundedness $H_{1 *}: L_{v}^{p} \rightarrow L_{u}^{q}$ does hold because of (3.10), (2.10) and (2.11).

C) Finally, we have to show that condition (2.9) and $H_{1 *}: L_{v}^{p} \rightarrow L_{u}^{q}$ lead to the discrete boundedness $\mathbf{H}_{1}: \ell_{v}^{p} \rightarrow \ell_{u}^{q}$.

Therefore Steps B) and C) yield the proof of the sufficient part in Theorem 2.2.

Acknowledgement. The author would like to thank the referee for having pointed him out that the idea to solve the discrete problem via the corresponding continuous one was previously used by G. Hardy, J. Littlewood and G. Pólya in [1].

\section{References}

[1] Hardy J., Littlewood J. E. and Pólya G.: Inequalities (Cambridge Mathematical Library). Cambridge: Univ. Press 1934.

[2] Hwang, D. Y.: A many variable generalization of the discrete Hardy's inequality. Tamkang J. Math. 27 (1996), $125-132$.

[3] Kamaly, A. and Y. Rakotondratsimba: Weighted inequalities for discrete and integral Hardy operators. SUT J. Math 33 (1997), 183 - 188.

[4] Kamaly, A. and Y. Rakotondratsimba: Weighted inequalities for two-dimensional discrete Hardy operators. Thesis by A. Kamaly. Stockholm: KTH 1998.

[5] Leindler, L.: On the converses of inequalities of Hardy and Littlewood. Acta Sci. Math (Szeged) 58 (1993), $191-196$.

[6] Németh, Z.: Inequalities of Hardy-Littlewood type for double series. Acta Sci. Math (Szeged) 62 (1993), 153 - 159.

[7) Pachpatte, B.: A many variable generalization of Hardy's inequality concerning a serie of terms. Tamkang J. Math. 23 (1992), 349 - 354. 
[8] Sawyer, E.: Weighted inequalities for the two-dimensional Hardy operator. Studia Math $82(1985), 1-16$.

Received 28.09.1998 